\title{
Application of Computer Vision for quality control in frozen mixed berries production: colour calibration issues
}

\author{
D. Ricauda Aimonino, P. Gay, L. Comba \\ DI.S.A.F.A, Università di Torino, Grugliasco (TO), Italy
}

\section{Abstract}

Computer vision is becoming increasingly important in quality control of many food processes. The appearance properties of food products (colour, texture, shape and size) are, in fact, correlated with organoleptic characteristics and/or the presence of defects. Quality control based on image processing eliminates the subjectivity of human visual inspection, allowing rapid and non-destructive analysis. However, most food matrices show a wide variability in appearance features, therefore robust and customized image elaboration algorithms have to be implemented for each specific product. For this reason, quality control by visual inspection is still rather diffused in several food processes. The case study inspiring this paper concerns the production of frozen mixed berries. Once frozen, different kinds of berries are mixed together, in different amounts, according to a recipe. The correct quantity of each kind of fruit, within a certain tolerance, has to be ensured by producers. Quality control relies on bringing few samples for each production lot (samples of the same weight) and, manually, counting the amount of each species. This operation is tedious, subject to errors, and time consuming, while a computer vision system (CVS) could determine the amount of each kind of berries in a few seconds. This paper discusses the problem of colour calibration of the CVS used for frozen berries mixture evaluation. Images are acquired by a digital camera coupled with a dome lighting system, which gives a homogeneous illumination on the entire visible surface of the berries, and a flat bed scanner. RBG device dependent data are then mapped onto CIELab colorimetric colour space using different transformation operators. The obtained results show that the proposed calibration procedure leads to colour discrepancies comparable or even below the human eyes sensibility.

Correspondence: Davide Ricauda Aimonino, Dipartimento di Scienze Agrarie, Forestali e Alimentari (DI.S.A.F.A.) Università degli Studi di Torino, via Leonardo da Vinci, 44, 10095 Grugliasco (T0), Italy.

Tel. +39.011.6708890, Fax +39.011 .6708591$

E-mail: davide.ricauda@unito.it

Key words: computer vision, colour calibration, food quality, frozen berries.

Acknowledgements: this work was partially supported by the grants of the project FoodVision (POR-FESR - Regione Piemonte).

(C) Copyright D. Ricauda Aimonino et al., 2013

Licensee PAGEPress, Italy

Journal of Agricultural Engineering 2013; XLIV(s2):e54

doi:10.4081/jae.2013.s2.e54

This article is distributed under the terms of the Creative Commons Attribution Noncommercial License (by-nc 3.0) which permits any noncommercial use, distribution, and reproduction in any medium, provided the original author(s) and source are credited.

\section{Introduction}

Over the last two decades, image processing has been rapidly diffused in food industry as instrument for automatic food quality evaluation and control (Du and Sun, 2004; Jackman et al., 2012). Computer Vision Systems (CVSs) can effectively replace visual (human) inspection in different contexts of food industry. Furthermore, they provide more objective and standard evaluation of some food quality parameters over a great number of samples (Du and Sun, 2004; K. León et al., 2006).

Most food products have a heterogeneous matrix; therefore their appearance properties (colour, texture, shape and size) can be strongly variable, even for the same product category. For this reason specific image processing tools have to be developed for every specific product as well as ad hoc image acquisition systems have to be implemented in same particular cases. These aspects are the main limitation to a large-scale deployment of CVS in food industry, in particular in small and medium enterprises, where quality control is mainly entrusted to trained inspectors.

Among food quality evaluations based on products appearance, one of the most repetitive and tedious is the quality control of frozen mixed fruits or vegetables. An example is the production of frozen mixed berries. Usually, berries are severally frozen and then mixed together, in different amounts, according to a recipe. Since producers have to ensure the correct quantity (within a certain tolerance) of each kind of fruit in the mixture, quality control consists in manually counting the amount of each of them in samples (with the same weight) collected by different production lots.

This task could be efficiently carried out by a CVS, analysing a whole product sample in few seconds.

The identification of different objects within a digital image of a food product is typically based on shape and colour appearance attributes, even if texture and size are also used in many cases (Du and Sun, 2004; C. Zheng et al., 2006; Jackman and Sun, 2013). Different classification methods can be adopted to classify the objects in a number of classes with similar appearance properties. Statistical, fuzzy logic and neural network are the most used ones (Du and Sun, 2004). While shape analysis can be usually performed adopting morphological operations available in image processing tools, colour measurement is a nontrivial task. Digital colour images are acquired in RGB colour space, which is not a colorimetric space (Hong et al., 2001; Green, 2003). RGB signals provided by an image acquisition system (camera, scanner) are device-dependent, i.e. different devices (even of the same type) can give different responses. Therefore, a colour space transform that maps RGB values of a digital image onto a device-independent colorimetric colour space, such as CIELab, has to be defined for accurate colour measurements (Hong et al., 2001; Westland and Ripamonti, 2004; K. León et al., 2006; Wu and Sun, 2013). This procedure is usually called device colour characterization.

A preliminary study about the development of a laboratory CVS for 
frozen mixed berries quality inspection is presented in this paper. In particular, the article is focused on the colour characterization of the imaging system. A target-based approach has been adopted, in which a set of colour samples, with known XYZ and/or Lab values, is considered as reference. Different polynomial transforms (from RGB to CIELab colour space) for colour characterization were compared and applied to two image acquisition systems: a flat bed scanner and a CVS based on a digital camera. Performances of the calibration models were evaluated in terms of colour measurement accuracy and calculation times.

\section{Image acquisition systems}

A simple way to classify and count fruits in a sample of frozen mixed berries is to spread them randomly on a flat surface painted with al light colour, in order to have a good contrast between berries and background in the digital image. This layout suggested to carried out tests collecting images both by a flat bed scanner and a CVS equipped with a digital camera.

In the first case an A4 Microtek ScanMaker i 900 flat bed scanner was adopted. Scan surface was covered with a wooden cover $(210 \mathrm{~mm} \times 290$ $\mathrm{mm} \times 50 \mathrm{~mm}$ ) to shield ambient light, whose internal walls were painted with a light grey paint, similar to the standard grey for cameras white balance (X-rite ColorChecker white balance card).

The CVS consisted of a Nikon D5100 ${ }^{\circledR}$ colour digital camera coupled with a dome lighting system, which ensured a uniform illumination of berries without shadows. A white plastic hemisphere $(350 \mathrm{~mm}$ diameter) reflects the light provided by two LEDs arrays, obtaining a colour temperature of about $5500 \mathrm{~K}$. The camera was mounted on a stand together the hemisphere as shown in Figure 1. Lifting up them, berries samples can be arranged within the circular surface defined by the LEDs support. Images acquisition and camera set up were remotely controlled by NKRemote ${ }^{\circledR}$ software (Breeze System - UK) installed on Laptop PC connected to the camera with the USB port. The camera and the illumination system were placed into a $(500 \mathrm{~mm} \times 600 \mathrm{~mm} \times 900$ $\mathrm{mm}$ ) wooden box internally painted black to avoid external light and reflections (Valous et al., 2009). A manual camera white balance was carried out, using an X-rite ColorChecker white balance card, before device characterization. A set of card images was collected changing the camera colour temperature parameter, until the three histograms of the RGB channels were exactly overlapped. The obtained value was adopted in all subsequent image acquisitions, maintaining the automatic white balance disabled as suggested by (Cheung et al., 2004).

\section{Devices colour characterization}

Device characterization mainly consists in to find one or more transforms between the device RGB values and an absolute device-independent, metric, colour space, usually CIELab. The result of this process is an image represented in $\mathrm{L}^{*} \mathrm{a}^{*} \mathrm{~b}^{*}$ colour coordinates on which colorimetric measurements can be performed. In target-based approach, an image of a reference colour card with a number of colour samples is acquired by the CVS to obtain their mean RGB values, while the correspondent XYZ values are measured by a spectrophotometer. Different methods can be applied to derive the RGB-XYZ transform: look up tables with interpolation, polynomial regressions and neural network among all (Hong et al., 2001; Wu and Sun, 2013). CIELab colour coordinates are then calculated applying the following standard transformation:

$$
\begin{aligned}
& L^{*}= \begin{cases}116\left(\frac{Y}{Y_{n}}\right)^{1 / 3}-16 & \text { if }\left(\frac{Y}{Y_{n}}\right)>0.008856 \\
903.3\left(\frac{Y}{Y_{n}}\right) & \text { if }\left(\frac{Y}{Y_{n}}\right) \leq 0.008856\end{cases} \\
& a^{*}=500\left[\left(\frac{X}{X_{n}}\right)^{1 / 3}-\left(\frac{Y}{Y_{n}}\right)^{1 / 3}\right] \\
& b^{*}=200\left[\left(\frac{Y}{Y_{n}}\right)^{1 / 3}-\left(\frac{Z}{Z_{n}}\right)^{1 / 3}\right]
\end{aligned}
$$

where $X_{n}, Y_{n}$ and $Z_{n}$ are the tristimulus values of a reference white for a certain CIE standard illuminant (Green, 2003).

A X-right ColorChecker passport (a poked-size version of traditional ColorChecker) was used as colour reference in present work. This card is a collection of $24(20 \mathrm{~mm} \times 20 \mathrm{~mm})$ coloured patches, which XYZ val-

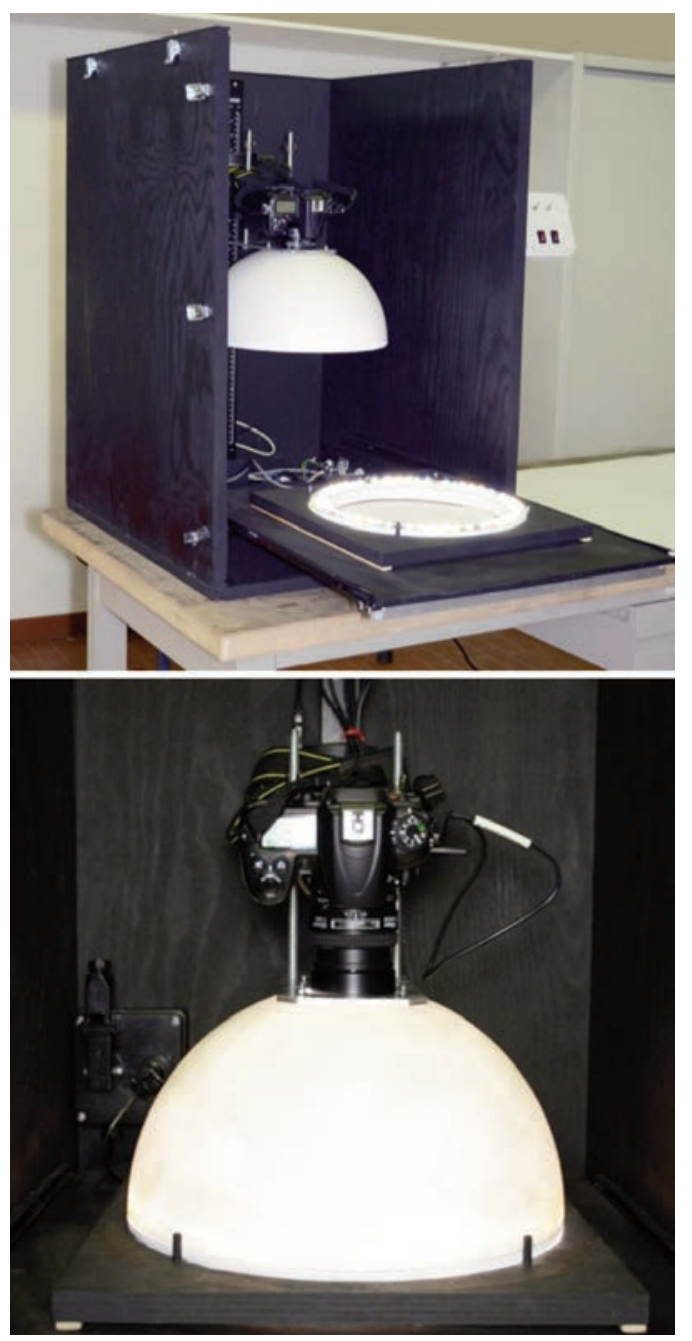

Figure 1. The Computer Vision System (without front and top walls) in which camera and the white hemisphere are lifted up to allow samples arrangement (on left); the same system during image acquisition (on right). 
ues of each patch are collected as a result of five subsequent measurements carried out with a X-rite spectrophotometer (D50 illuminant, $2^{\circ}$ observer). Digital images of the ColorChecker were acquired by the scanner and the CVS to obtain the input devices RGB values of the 24 coloured patches. These values were calculated as the average of the RGB values of all pixels corresponding to each colour patch, excluding boundary pixels. CEI tristimulus values (XYZ) of ColorChecker card and the corresponding device RBG ones were used to carry out colour characterization considering different polynomial regression models with least-squares fitting. The transform from RGB device values to $\mathrm{XYZ}$ can be performed applying the following relationship to each pixel of an image:

$$
\left[\begin{array}{l}
X \\
Y \\
Z
\end{array}\right]=C p
$$

where $C$ is the $(3 \times n)$ calibration matrix calculated with the last squares fitting polynomial regression (see e.g. (Hong et al., 2001) for a detailed decryption of the method), and $p$ is a vector whose $n \times 1$ elements are the terms of the correspondent polynomial calculated with the $r, g$, and $b$ normalized values of the pixel. The following polynomials were compared in this paper:

$\begin{array}{lll} & \text { Calibration Model } & n \\ p_{1} & r g b & 3 \\ p_{2} & r g b 1 & 4 \\ p_{3} & r g b r g b & 4 \\ p_{4} & r g b r g b 1 & 5 \\ p_{5} & r g b r g r b g b & 6 \\ p_{6} & r g b r^{2} g^{2} b^{2} & 6 \\ p_{7} & r g b r g r b g b 1 & 7 \\ p_{8} & r g b r g r b g b r g b & 7 \\ p_{9} & r g b r^{2} g^{2} b^{2} 1 & 7 \\ p_{10} & r g b r^{2} g^{2} b^{2} r g b & 7 \\ p_{11} & r g b r g r b g b r g b 1 & 8 \\ p_{12} & r g b r^{2} g^{2} b^{2} r g b 1 & 10 \\ p_{13} & r g b r^{2} g^{2} b^{2} r g r b g b & 10 \\ p_{14} & r g b r^{2} g^{2} b^{2} r g r b g b 1 & 11 \\ p_{15} & r g b r^{2} g^{2} b^{2} r g r b g b r g b & 14 \\ p_{16} & r g b r^{2} g^{2} b^{2} r g r b b r g b 1 & 17 \\ p_{17} & r g b r^{3} g^{3} b^{3} r^{2} g^{2} b^{2} r g r b g b r g b 1 & 20 \\ p_{18} & r g b r^{4} g^{4} b^{4} r^{3} g^{3} b^{3} r^{2} g^{2} b^{2} r g r b g b r g b 1 & 20 \\ p_{19} & r g b r^{5} g^{5} b^{5} r^{4} g^{4} b^{4} r^{3} g^{3} b^{3} r^{2} g^{2} b^{2} r g r b g b r g b 1 & 23 \\ p_{20} & r g b r g r b g b r^{2} g^{2} b^{2} r g b r^{2} g g^{2} b b^{2} r r^{2} b g^{2} r b^{2} g r^{3} g^{3} b^{3} 1 & \\ p_{21} & r g b r^{6} g^{6} b^{6} r^{5} g^{5} b^{5} r^{4} g^{4} b^{4} r^{3} g^{3} b^{3} r^{2} g^{2} b^{2} r g r b g b r g b 1 & \end{array}$

However, usually, colour characterization cannot be directly applied to normalized RGB values of an image, because most image acquisition devices show nonlinearities between the input light intensity and the response of the colour channels. Although CCD sensors are characterized by a linear response to light intensity, a nonlinearity was added by manufacturers according to sRGB standard (gamma correction). Therefore a linearization process is recommended before applying colour characterizations transformations, even in the case of polynomial (nonlinear) models (Cheung et al., 2004). The linearization consists in to find the inverse function of added nonlinearity. Luminance (Y coordinate of XYZ colour space) and reflectance of grey colour samples are almost constant within the visible light spectrum; therefore RGB channels response is described by the trend of these parameters depending on RGB values obtained for the same grey samples (Westland and Ripamonti, 2004). Figures 2 and 3 show the nonlinear relationships between RGB responses and mean reflectance of the six ColorChecker grey samples for CVS and scanner respectively. The same behaviour is shown for luminance (not reported). These relationships represent the linearization functions for $\mathrm{R}, \mathrm{G}$ and $\mathrm{B}$ channel, which can be calculated by least-square polynomial regression. Four polynomial (3-rd, 4-th, 5-th and 6-th degree) fitting are tested evaluating their effect on scanner and CVS colour characterization. The measure of the colour difference between the values estimated by colour characterization (, , and ) and those measured with spectrophotometer (, , and ) was calculated, for the 24 ColorChecker patches.

$$
\Delta E=\sqrt{\left(L_{e}^{*}-L_{m}^{*}\right)^{2}+\left(a_{e}^{*}-a_{m}^{*}\right)^{2}+\left(b_{e}^{*}-b_{m}^{*}\right)^{2}}
$$

Calculation times for linearization and colour characterization procedures are also calculated as performance index, adopting $1280 \times 848$ pixels images as reference.

All algorithms for channel response linearization, colour characterization and results comparison were implemented with Matlab ${ }^{\circledR}$ (MathWorks, USA).

\section{Results}

CVS camera and scanner channels responses (Figures 2 and 3 ) show a power law trend, typical of gamma corrections. Increasing the order of the polynomial fitting for channel response linearization, $R^{2}$ regression coefficient increases as well as calculation times (Table 1), denot-
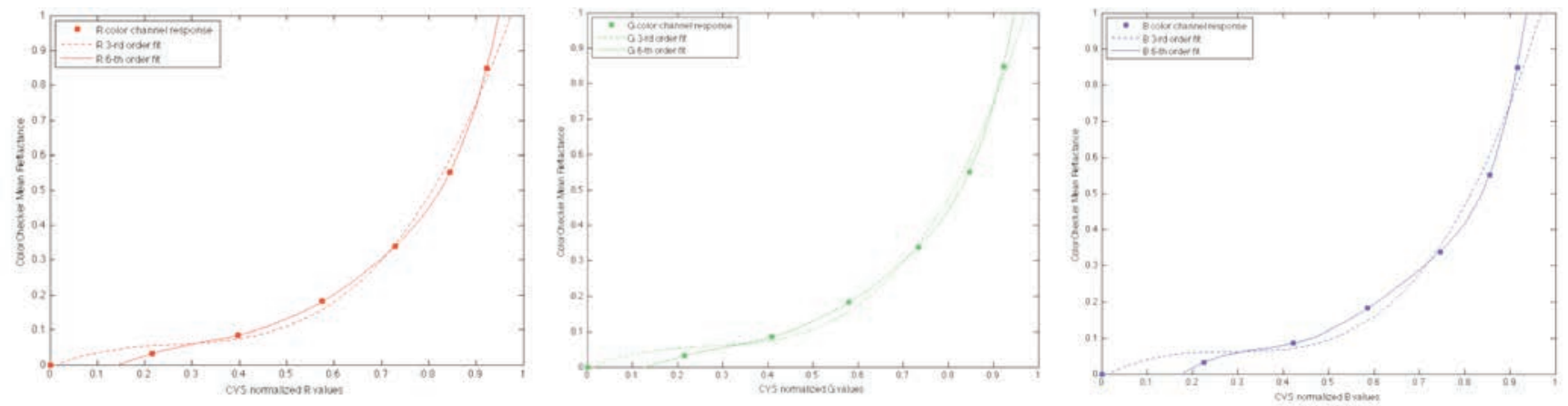

Figure 2. CVS camera RGB channels response (relationships between normalized RGB values of ColorChecker grey patches and Mean Reflectance), and corresponding 3-rd and 6-th order linearization polynomial fits. 

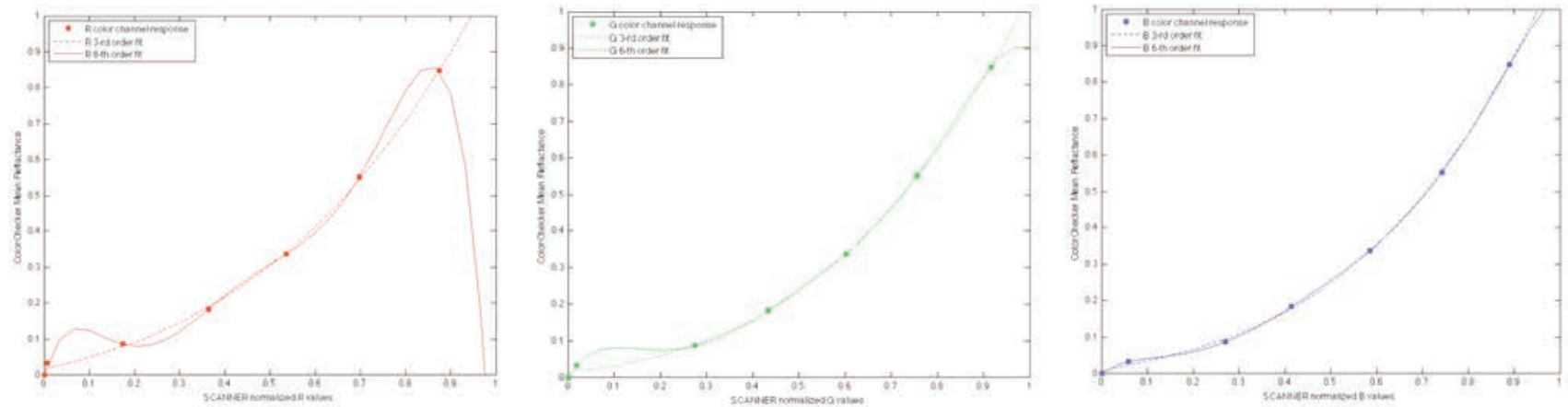

Figure 3. Scanner RGB channels response (relationships between normalized RGB values of ColorChecker grey patches and Mean Reflectance), and corresponding 3-rd and 6-th order linearization polynomial fits.

Table 1. Regression coefficients $\left(R^{2}\right)$ and calculation times for $R, G$ and $B$ channels linearization (for 3-rd, 4-th, 5-th and 6-th degree polynomials models) adopting mean reflectance and luminance.

\begin{tabular}{|c|c|c|c|c|c|c|c|c|}
\hline \multirow[b]{2}{*}{ Image acquisition system } & \multirow[b]{2}{*}{ Polynomial degree } & \multirow[b]{2}{*}{ Calculation time [ms] } & \multicolumn{3}{|c|}{ Mean Reflectance } & \multicolumn{3}{|c|}{ Luminance } \\
\hline & & & $\mathbf{R}_{\mathrm{R}}^{2}$ & $\mathbf{R}_{6}^{2}$ & $\mathrm{R}_{\mathrm{B}}^{2}$ & $\mathbf{R}_{\mathrm{R}}^{2}$ & $\mathrm{R}_{\mathrm{G}}^{2}$ & $\mathbf{R}_{\mathrm{B}}^{2}$ \\
\hline \multirow[t]{4}{*}{ CVS } & 3 & 138 & 0.9936 & 0.9930 & 0.9863 & 0.9828 & 0.9822 & 0.9755 \\
\hline & 4 & 146 & 0.9987 & 0.9985 & 0.9958 & 0.9879 & 0.9876 & 0.9850 \\
\hline & 5 & 154 & 0.9998 & 0.9998 & 0.9993 & 0.9889 & 0.9890 & 0.9885 \\
\hline & 6 & 165 & 1.0000 & 1.0000 & 1.0000 & 0.9891 & 0.9891 & 0.9891 \\
\hline \multirow[t]{4}{*}{ Scanner } & 3 & 138 & 0.9995 & 0.9998 & 0.9997 & 0.9886 & 0.9889 & 0.9888 \\
\hline & 4 & 146 & 0.9998 & 0.9999 & 0.9997 & 0.9889 & 0.9890 & 0.9888 \\
\hline & 5 & 154 & 0.9999 & 0.9999 & 0.9999 & 0.9890 & 0.9890 & 0.9890 \\
\hline & 6 & 165 & 1.0000 & 1.0000 & 1.0000 & 0.9891 & 0.9891 & 0.9891 \\
\hline
\end{tabular}

Table 2. Performances of CVS colour characterization polynomial models with different mean reflectance linearization functions (3-rd, 4-th, 5-th and 6th degree polynomials fitting).

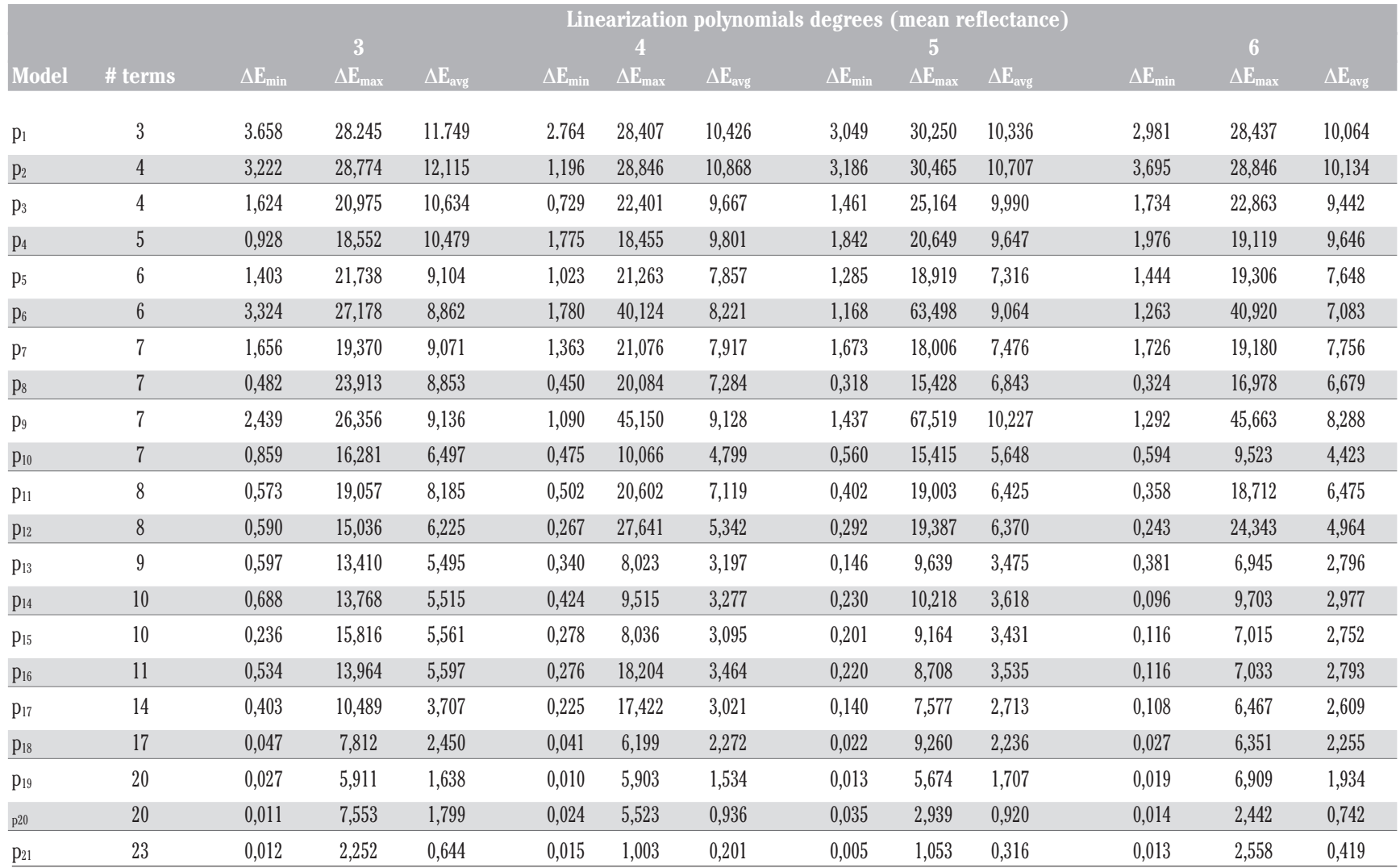


Table 3. Performances of p19, p20 and p21 colour characterization models with different linearization functions (3-rd, 4-th, 5-th and 6-th degree polynomials fitting).

\begin{tabular}{|c|c|c|c|c|c|c|c|c|c|c|c|c|c|}
\hline & \multicolumn{13}{|c|}{ Linearization polynomials degrees (mean reflectance) } \\
\hline & \multirow{2}{*}{$\begin{array}{c}\text { Model } \\
\text { \# terms }\end{array}$} & \multicolumn{3}{|c|}{3} & \multicolumn{3}{|c|}{4} & \multicolumn{3}{|c|}{5} & \multicolumn{3}{|c|}{6} \\
\hline & & $\Delta \mathbf{E}_{\min }$ & $\Delta \mathbf{E}_{\max }$ & $\Delta \mathrm{E}_{\text {avg }}$ & $\Delta \mathbf{E}_{\min }$ & $\Delta \mathbf{E}_{\max }$ & $\Delta \mathbf{E}_{\text {avg }}$ & $\Delta \mathrm{E}_{\min }$ & $\Delta \mathbf{E}_{\max }$ & $\Delta \mathrm{E}_{\text {avg }}$ & $\Delta \mathbf{E}_{\min }$ & $\Delta \mathrm{E}_{\max }$ & $\Delta \mathrm{E}_{\text {avg }}$ \\
\hline \multicolumn{14}{|l|}{ CVS } \\
\hline Mean & $\mathrm{p}_{19}(20)$ & 0,027 & 5,911 & 1,638 & 0,001 & 5,903 & 1,534 & 0,013 & 5,674 & 1,707 & 0,019 & 6,909 & 1,934 \\
\hline \multirow[t]{2}{*}{ Refl. } & $\mathrm{p}_{20}(20)$ & 0,011 & 7,553 & 1,799 & 0,024 & 5,523 & 0,936 & 0,035 & 2,939 & 0,92 & 0,014 & 2,442 & 0,742 \\
\hline & $\mathrm{p}_{21}(23)$ & 0,012 & 2,252 & 0,644 & 0,015 & 1,003 & 0,201 & 0,005 & 1,053 & 0,316 & 0,013 & 2,558 & 0,419 \\
\hline \multirow[t]{3}{*}{ Lum. } & p19 (20) & 0,020 & 7,004 & 1,963 & 0,009 & 7,011 & 1,634 & 0,012 & 8,800 & 2,074 & 0,016 & 7,302 & 2,156 \\
\hline & p20 (20) & 0,009 & 7,721 & 1,847 & 0,021 & 5,487 & 0,927 & 0,033 & 3,356 & 1,280 & 0,011 & 3,097 & 0,789 \\
\hline & p21 (23) & 0,009 & 2,333 & 0,626 & 0,023 & 1,773 & 0,406 & 0,007 & 1,540 & 0,291 & 0,015 & 3,347 & 0,589 \\
\hline \multicolumn{14}{|l|}{ Scanner } \\
\hline Mean & $\mathrm{p}_{19}(20)$ & 0,029 & 6,065 & 1,848 & 0,017 & 5,939 & 1,857 & 0,020 & 5,857 & 1,530 & 0,028 & 10,028 & 2,837 \\
\hline \multirow[t]{2}{*}{ Refl. } & $\mathrm{p}_{20}(20)$ & 0,021 & 3,395 & 0,860 & 0,022 & 3,701 & 0,844 & 0,021 & 3,810 & 0,886 & 0,036 & 11,739 & 2,696 \\
\hline & $\mathrm{p}_{21}(23)$ & 0,012 & 4,062 & 0,774 & 0,013 & 3,436 & 0,672 & 0,015 & 3,757 & 0,906 & 0,008 & 8,882 & 1,378 \\
\hline \multirow[t]{3}{*}{ Lum. } & $\mathrm{p}_{19}(20)$ & 0,026 & 6,081 & 1,864 & 0,015 & 5,987 & 1,879 & 0,019 & 5,973 & 1,517 & 0,028 & 10,087 & 2,775 \\
\hline & $\mathrm{P}_{20}(20)$ & 0,020 & 3,304 & 0,850 & 0,022 & 3,526 & 0,801 & 0,023 & 3,892 & 0,888 & 0,028 & 10,413 & 2,432 \\
\hline & $\mathrm{p}_{21}(23)$ & 0,012 & 4,190 & 0,784 & 0,012 & 3,672 & 0,711 & 0,014 & 3,796 & 0,939 & 0,008 & 8,209 & 1,282 \\
\hline
\end{tabular}

ing a better fitting. Linearization with luminance gives slightly lower $\mathrm{R}^{2}$ values than the case with mean reflectance for both acquisition systems, even if the polynomial fitting seems to perform better for the flat bed scanner.

Regarding to colour characterization (Table 2) colour differences between values estimated by the different models and measured values generally decreases increasing the number of polynomial terms, even if this is not always the case. According to Hong et al. (2001), models performances depend on the terms used. Considering colour differences obtained with 7 terms polynomial models, better performances of $p_{10}$ respect to the other models $\left(p_{7}, p_{8}, p_{9}\right)$ can be observed regardless the adopted linearization fitting. In particular the term $r g b$ determines lower $\Delta \mathrm{E}$ values respect to the term 1 , as can also be noted for $p_{2}$ and $p_{3}$ models.

However, in order to obtain accurate colour measurements, average $\Delta \mathrm{E}$ should be less than 2.2 , since this is considered the minimum value of colour difference distinguishable by human eyes (Valous et al., $2009)$. For this reason only the last three models $\left(p_{19}, p_{20}, p_{21}\right)$ were compared for CVS camera and scanner considering the different linearization polynomial fittings. A progressive reduction of $\Delta \mathrm{E}$ values was observed increasing the degree of the linearization polynomial fitting for CVS, using both mean reflectance and luminance (Table 2 and $3)$. Colour characterization performances for the scanner show a different behaviour, with an initial reduction of $\Delta \mathrm{E}$, adopting 3-rd and 4-th degree polynomials, and a subsequent colour difference increase with higher order polynomials. This behaviour can be explained comparing the shape of approximating polynomials graphs in Figures 2 and 3. Note that, both polynomial fits are increasing functions in the case of CVS (Figure 2), moreover 6-th order polynomial fit provides a better fitting than the 3-th one. On the contrary, 3-th and 6-th order approximating polynomials have different behaviours for scanner linearization. The first one is an increasing function, which mind a typical gamma correction, whereas the 6 -th order polynomial fit shows a relative and an absolute maximum for $\mathrm{R}$ and $\mathrm{G}$ channels. This means that in this case polynomial fit does not approximate a gamma correction curve correctly, in spite of $\mathrm{R}^{2}$ of the 6 -th order $\mathrm{R}$ and $\mathrm{G}$ channels polynomial fits are greater than the 3 -th order ones. Therefore, high $\mathrm{R}^{2}$ values in linearization process do not necessarily correspond to better colour characterization performances.

It also important to note that CVS provides better performances than

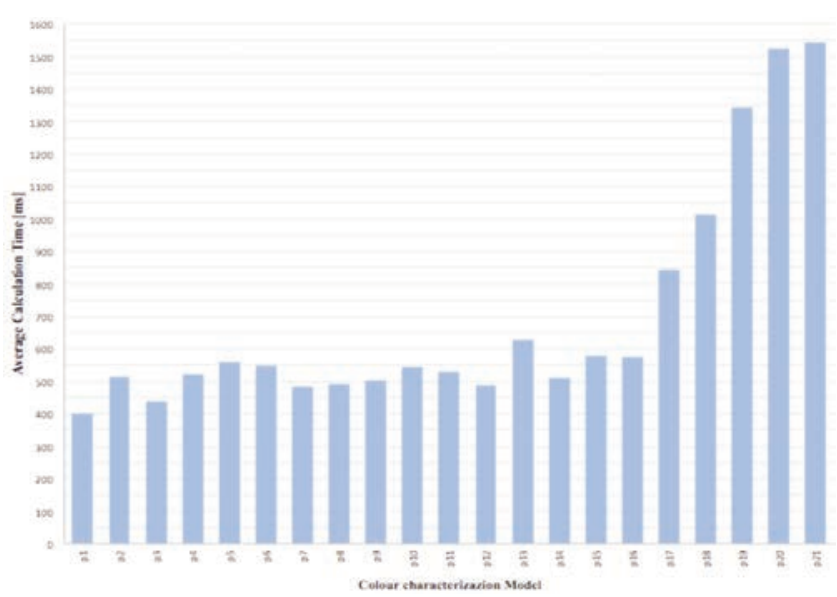

Figure 4. Average calculation times of colour characterization process for the adopted models.

the scanner (Table 3). This difference can be attribute to the white balance performed for CVS camera that probably improves its accuracy.

Average calculation times were evaluated for all colour characterization models, applying the characterization algorithm to ten different images $(1280 \times 848)$. Data reported in Figure 4 show that processing times are absolutely compatible with laboratory analyses even adopting high performances models (about 1.5 seconds).

\section{Conclusions}

Colour characterization of an imaging system is an essential step for colour measurements on food products. Food quality controls, based on products appearance properties, require accurate colour measurements to adequately detect defects and/or products classification. Polynomial characterization models, preceded by a RGB channel response linearization, can achieve high accuracy with reasonable cal- 
culation times. Therefore this technique can be profitably employed in laboratory applications. Approximating polynomials can be adopted even in channel response linearization process. However, increasing the order of the polynomial fits do not generally correspond to a performance improvement of the whole colour characterization. Therefore this issue has to be taken in account if an automatic calibration tool is developed.

Further investigations about colour characterization will be carried out evaluating models performances in berries classification in order to look for the best trade of between classification accuracy and calculation times.

\section{References}

Cheung, V., Westland, S., Thomson, M. 2004. Accurate estimation of the nonlinearity of input/output response for color cameras. Color Res. Appl. 29: 406-412.

Du, C.-J., Sun, D.-W. 2004. Recent developments in the applications of image processing techniques for food quality evaluation. Trends Food Sci. Technol. 15: 230-249.

Green, P. 2003. Colorimetry and colour difference, in: Colour Engineering Achieving Device Independent Colour, Display
Technology. John Wiley \& sons, 49-77.

Hong, G., Luo, M.R., Rhodes, P.A. 2001. A study of digital camera colorimetric characterization based on polynomial modeling. Color Res. Appl. 26: 76-84

Jackman, P., Sun, D.-W. 2013. Recent advances in image processing using image texture features for food quality assessment. Trends Food Sci. Technol. 29: 35-43.

Jackman, P., Sun, D.-W., ElMasry, G. 2012. Robust colour calibration of an imaging system using a colour space transform and advanced regression modelling. Meat Sci. 91: 402-407.

León, K., Mery, D., Pedreschi, F., León, J. 2006. Color measurement in L a b units from RGB digital images. Food Res. Int. 39: 1084-1091.

Valous, N.A., Mendoza, F., Sun, D.-W., Allen, P. 2009. Colour calibration of a laboratory computer vision system for quality evaluation of presliced hams. Meat Sci. 81: 132-141.

Westland, S., Ripamonti, C. 2004. Characterization of Cameras, in: Computational Color Science Using Matlab. Wiley, 127-140.

Wu, D., Sun, D.-W. 2013. Colour measurements by computer vision for food quality control - A review. Trends Food Sci. Technol. 29: 5-20.

Zheng, C., Sun, D.-W., Zheng, L. 2006. Recent developments and applications of image features for food quality evaluation and inspection - a review. Trends Food Sci. Technol. 17: 642-655. 